\title{
Nucleon and Delta Sigma-Terms
}

\author{
I. P. Cavalcante, ${ }^{1}$ M. R. Robilotta, ${ }^{2}$ J. Sá Borges, ${ }^{3}$ D. de O. Santos, ${ }^{1}$ and G. R. S. Zarnauskas ${ }^{2}$ \\ ${ }^{I}$ Depto. de Física, CCET, Universidade Federal de Mato Grosso do Sul, \\ C.P. 549, C.E.P. 79070-900, Campo Grande, MS, Brazil. \\ ${ }^{2}$ Instituto de Física, Universidade de São Paulo, \\ C.P. 66318, 05315-970, São Paulo, SP, Brazil. \\ ${ }^{3}$ Universidade do Estado do Rio de Janeiro, Instituto de Física, \\ Rua São Francisco Xavier, 524, Maracanã, Rio de Janeiro, RJ, Brazil. \\ Received on 29 September, 2006
}

\begin{abstract}
We evaluate nucleon and delta sigma-terms and obtain the results $43 \mathrm{MeV} \leq \sigma_{N} \leq 49 \mathrm{MeV}$ and $28 \mathrm{MeV} \leq$ $\sigma_{\Delta} \leq 32 \mathrm{MeV}$, depending on the coupling constants used, which are compatible with values extracted from experiment and produced by other groups. We show that the decay $\Delta \rightarrow \pi N$ explains the relation $\sigma_{\Delta}<\sigma_{N}$.
\end{abstract}

Keywords: Nucleon and delta sigma terms; Delta decay; Effective theories

\section{INTRODUCTION}

QCD is nowadays the main theoretical framework to describe hadronic interactions, but its non-Abelian character makes calculations in the low-energy regime unfeasible. In order to overcome this problem, one may work with either lattice QCD or effective field theories.

Effective theories are constructed by writing the most general Lagrangian containing all the terms allowed by the symmetries of the main theory. In the case of chiral symmetry, the various terms are organized considering the number of pion masses or derivatives of the pion field. This procedure gives meaning to the idea of chiral perturbation theory (ChPT). Considering only $u$ and $d$ quarks, we assume that the effective theory possesses approximate $S U(2) \times S U(2)$ chiral symmetry, broken by the small pion mass in the effective theory. An important aspect of chiral dynamics concerns the effects of this breaking of the symmetry over the nucleon and delta resonance masses. The answer to this question is related to sigma-terms.

The delta $(\Delta)$ plays a very important role in low-energy pion-nucleon $(\pi N)$ scattering and correlated processes, such as the nucleon-nucleon interaction. When the delta is present, the scale of some amplitude denominators is given by the quantity $\omega_{\Delta} \sim M-m$. Delta contributions are given by ratios of small quantities and may turn out to be large. In such cases, numerical values adopted for $M-m$ do influence predictions produced by effective theories, especially those that rely on the small scale expansion [1] or the heavy baryon approximation [2].

In ChPT, there is a clear conceptual distinction between the bare baryon masses, present in the Lagrangian, and their respective observed values, which include loop corrections. The former should, in principle, be preferred as inputs in the evaluation of theoretical amplitudes. Nevertheless, as there is little information available concerning the bare delta mass, one tends to use physical values in calculations. In most cases, it is reasonable to expect that this would have little numerical importance. On the other hand, in the case of the parameter $\omega_{\Delta}$, which is a small quantity, the influence of loop corrections may become relatively large.

According to the Feynman-Hellmann theorem [3] the mass
$m_{B}$ of a baryon $B$ is related to its sigma-term $\sigma_{B}$ by $\sigma_{B}=$ $\mu^{2} d m_{B} / d \mu^{2}$. Therefore the sigma-term provides a measure of the shift in the baryon mass due to chiral symmetry breaking. Whenever it is possible to evaluate $\sigma_{B}$ as a function of $\mu$, the bare mass $m_{B_{0}}$ can be extracted from the relation

$$
m_{B}=m_{B_{0}}+\int_{0}^{\mu^{2}} d \lambda \sigma_{B}(\lambda) / \lambda
$$

As the leading term in $\sigma_{B}$ is proportional to $\mu^{2}$, the difference $m_{B}-\sigma_{B}$ provides a crude estimate for $m_{B_{0}}$. In the case of the nucleon, one has $\sigma_{N}=45 \mathrm{MeV}$ [4]. In ChPT, the leading contribution to $\sigma_{N}$ cannot be predicted theoretically. Formally, it is associated with the constant $c_{1}$ of the second order Lagrangian $[5,6]$, which can be extracted from empirical subthreshold information. The situation of the delta is much worse, for $\pi \Delta$ scattering data are not available. One is then forced to resort to models in order to calculate the delta sigma-term, which is associated with the parameter $a_{1}$ defined in ref. [1].

This paper is organized as follows. In section II we review our evaluation of the nucleon and delta sigma-terms, presented in [7] (the reader is referred to this paper for a detailed description of the work). In section III we show that the relation $\sigma_{\Delta}<\sigma_{N}$ is associated with the fact that the delta can decay. A brief summary is provided in section IV.

\section{NUCLEON AND DELTA SIGMA-TERMS}

Th nucleon sigma-term obtained by applying ChPT at $O\left(q^{4}\right)$ depends on the low-energy constants (LEC's) $c_{1}, c_{2}$ and $c_{3}$. In this work we review a model presented in $[7,8]$.

Data on $\pi N$ subthreshold coefficients indicate that $c_{2}$ and $c_{3}$ are larger than $c_{1}$ and that their values are approximately saturated by $\Delta$ intermediate states [6]. Thus, up to $O\left(q^{4}\right)$, the function $\sigma_{N}(t)$ can be well represented by the leading tree contribution associated with $c_{1}$, supplemented by the two triangle diagrams shown in Fig.1, involving $N$ and $\Delta$ intermediate states.

The nucleon scalar form factor in momentum space is de- 


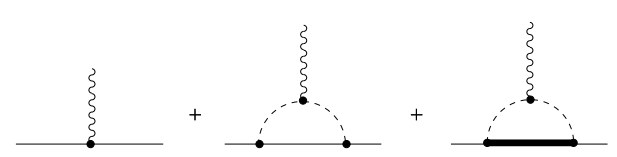

FIG. 1: Contact term and triangle diagrams contributing to the sigmaterm.

fined by

$$
\left\langle N\left(p^{\prime}\right)\left|-\mathcal{L}_{s b}\right| N(p)\right\rangle=\sigma_{N}(t) \bar{u}\left(p^{\prime}\right) u(p),
$$

where $\mathcal{L}_{s b}$ is the symmetry breaking term and $t=\left(p^{\prime}-p\right)^{2}$. In terms of the quark degrees of freedom, one has $\mathcal{L}_{s b}=$ $-\hat{m}(\bar{u} u+\bar{d} d)$, with $\hat{m}=\left(m_{u}+m_{d}\right) / 2$. The configuration space scalar form factor is denoted by $\tilde{\sigma}_{N}$ and given by

$$
\tilde{\sigma}_{N}(r)=\int \frac{d^{3} q}{(2 \pi)^{3}} e^{-i q \cdot r} \sigma_{N}(t),
$$

with $q=\left(p^{\prime}-p\right)$, in the Breit frame. The nucleon sigma-term, $\sigma_{N}$, is given by

$$
\sigma_{N}=4 \pi \int_{0}^{\infty} d r r^{2} \tilde{\sigma}_{N}(r)
$$

The contributions from the diagrams of Fig. 1 to $\tilde{\sigma}_{N}(r)$ read

$$
\tilde{\sigma}_{N}(r)=-4 c_{1} \mu^{2} \delta^{3}(r)+\tilde{\sigma}_{N_{N}}(r)+\tilde{\sigma}_{N_{\Delta}}(r),
$$

where $\tilde{\sigma}_{N_{N}}(r)$ and $\tilde{\sigma}_{N_{\Delta}}(r)$ denote contributions from diagrams with nucleon and delta as intermediate states, respectively.

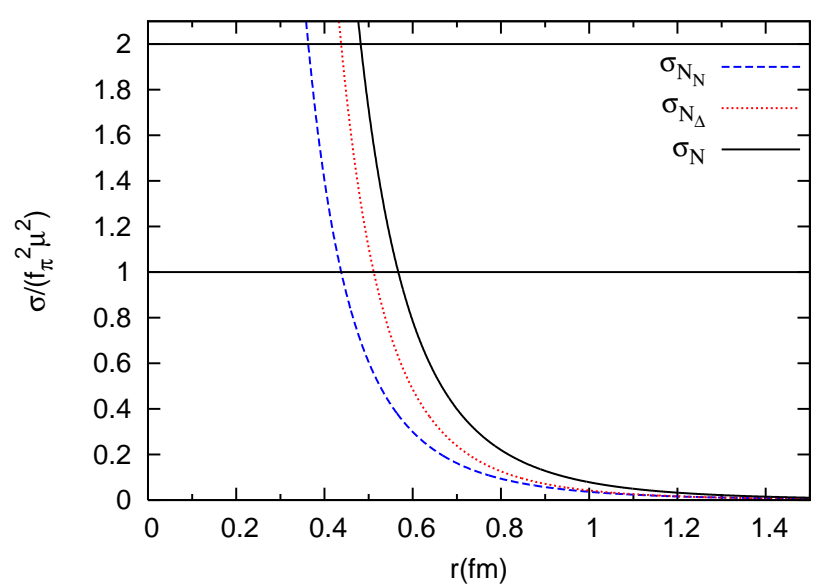

FIG. 2: Spatial dependence of the nucleon scalar form factor (solid curve) and partial contributions due to $N$ and $\Delta$ intermediate states (dashed curve and dotted curve, respectively).

In Fig.2, the condensed (QCD vacuum) and empty-space phases are represented by the horizontal lines at values 0 and 1 , respectively, whereas the curve $\sigma_{N}$ represents the influence of the nucleon over the condensate. At a critical point $R$ around $0.6 \mathrm{fm}$, this curve intersects the horizontal line at 1 , indicating the possibility of a phase transition. We assume that this phase transition does take place at this point and that the condensate no longer exists in the region $r<R$.
For this reason, in our previous evaluation of $\sigma_{N}[7,8]$, we used the expression

$$
\sigma_{N}=\frac{4}{3} \pi R^{3} f_{\pi}^{2} \mu^{2}+4 \pi \int_{R}^{\infty} d r r^{2} \tilde{\sigma}_{N}(r)
$$

instead of eq. (4). This procedure is the basis of our model and is more extensively discussed in ref. [7].

In the numerical determination of $\sigma_{N}$, we consider three possibilities for the $\pi N \Delta$ coupling constant. The corresponding results, given in table 1 , are quite close to the value extracted from experiment by Gasser, Leutwyler and Sainio [4], namely, $\sigma_{N}=45 \mathrm{MeV}$.

TABLE I: Nucleon sigma-term as a function of the $\pi N \Delta$ coupling constant.

\begin{tabular}{|c|c|c|}
\hline$g_{\pi N \Delta}$ & $R(\mathrm{fm})$ & $\sigma_{N}(\mathrm{MeV})$ \\
\hline \hline 1.19 & 0.55 & 42.6 \\
\hline 1.33 & 0.57 & 45.8 \\
\hline 1.47 & 0.59 & 49.4 \\
\hline
\end{tabular}

The delta scalar form factor is defined as

$$
\begin{aligned}
\left\langle\Delta\left(p^{\prime}, s^{\prime}\right)\left|-\mathcal{L}_{s b}\right| \Delta(p, s)\right\rangle \equiv & -\bar{u}_{\mu}^{s^{\prime}}\left(p^{\prime}\right)\left[g^{\mu \nu} \sigma_{\Delta}(t)\right. \\
& \left.+p^{\prime \nu} p^{\mu} F_{T}(t)\right] u_{v}^{s}(p),
\end{aligned}
$$

where $u_{v}^{s}$ is the $\Delta$ spinor [9] and $\sigma_{\Delta}$ and $F_{T}$ are, respectively, the scalar and tensor form factors. The minus sign on the r.h.s. is associated with the conventions used in the free $\Delta$ Lagrangian as in ref. [1]. We assume that the scalar form factor is determined by a short range contact interaction and two long range two-pion processes as in the case of the nucleon.

The values of the distance $R$ for which $\tilde{\sigma}_{\Delta}(R) / f_{\pi}^{2} \mu^{2}=1$ and the values of the delta sigma-term are given in table II, for different choices of the coupling constants $g_{\pi N \Delta}$ and $g_{\pi \Delta \Delta}$. Results for the real component of $\sigma_{\Delta}$ are sensitive to the coupling constant $g_{\pi \Delta \Delta}$ and consistent with that given in ref. [10], namely $\sigma_{\Delta}=(32 \pm 3) \mathrm{MeV}$. On the other hand, our prediction is larger than that quoted in ref. [11].

TABLE II: Partial contributions to $\sigma_{\Delta}$.

\begin{tabular}{|l|c||c|c|c|c|c|}
\hline$g_{\pi N \Delta}$ & $g_{\pi \Delta \Delta}$ & $R$ & core & cloud $N$ & cloud $\Delta$ & sum \\
\hline \hline 1.19 & 0.75 & 0.53 & 13.4 & -0.9 & 19.8 & 32.3 \\
\hline 1.19 & 0.67 & 0.51 & 12.0 & -0.4 & 16.6 & 28.2 \\
\hline 1.33 & 0.75 & 0.54 & 14.3 & -1.5 & 19.3 & 32.1 \\
\hline 1.33 & 0.67 & 0.52 & 12.9 & -0.9 & 16.1 & 28.1 \\
\hline 1.47 & 0.75 & 0.55 & 15.4 & -2.3 & 18.6 & 31.7 \\
\hline 1.47 & 0.67 & 0.53 & 14.0 & -1.7 & 15.5 & 27.8 \\
\hline
\end{tabular}

The structure of partial contributions for $S U(4)$ coupling constants, namely, $g_{\pi N \Delta}=1.33$ and $g_{\pi \Delta \Delta}=0.75$. is given in table III, where core and cloud refer to regions inside and outside the cutting radius $R$, respectively. 
TABLE III: Partial contributions to $\sigma_{N}$ and $\sigma_{\Delta}$.

\begin{tabular}{|l|c|c|c|c|}
\hline & core & cloud $N$ & cloud $\Delta$ & sum \\
\hline \hline$\sigma_{N}(\mathrm{MeV})$ & 16.7 & 13.0 & 16.1 & 45.8 \\
\hline$\sigma_{\Delta}(\mathrm{MeV})$ & 14.3 & -1.5 & 19.3 & 32.1 \\
\hline
\end{tabular}

\section{LOOP INTEGRALS}

The results given in table III show that the difference between $\sigma_{N}$ and $\sigma_{\Delta}$ is related to the cloud $N$ contribution to $\sigma_{\Delta}$, which is rather small, compared to $\sigma_{N}$. The reason for this behaviour is associated with the fact that the decay $\Delta \rightarrow \pi N$ is possible.

The decay $\Delta \rightarrow \pi N$ changes the behaviour of the loop integrals. In order to illustrate these changes and show the origin of the small size of the cloud $N$ contribution to $\sigma_{\Delta}$, we present some details of how we deal with the loop integrals.

In the evaluation of the cloud $N$ component of $\sigma_{\Delta}$, the following loop integral appears, among others:

$$
\begin{aligned}
I_{x \pi \pi}^{\mu \nu}= & \int \frac{d^{4} Q}{(2 \pi)^{4}} \frac{\left(Q^{\mu} Q^{v} / \mu^{2}\right)}{\left[(Q+q / 2)^{2}-\mu^{2}\right]\left[(Q-q / 2)^{2}-\mu^{2}\right]} \\
& \times \frac{2 \mu m_{e}}{\left[(Q+P)^{2}-m_{x}^{2}\right]}=\frac{i}{(4 \pi)^{2}}\left[g^{\mu v} \bar{\Pi}_{x \pi \pi}^{(000)}+\cdots\right],
\end{aligned}
$$

where $m_{e}$ and $m_{x}$ are the external and the internal baryon masses, respectively. We employ the variables

$$
q=\left(p-p^{\prime}\right), \quad P=\left(p+p^{\prime}\right) / 2, \quad Q=\left(k+k^{\prime}\right) / 2,
$$

where $p$ and $p^{\prime}$ are the initial and final baryon momenta, respectively, whereas $k$ and $k^{\prime}$ are the momenta of the exchanged pions.

The Feynman techniques for loop integration allow one to write the regular part of (8) as

$$
\begin{aligned}
\bar{\Pi}_{x \pi \pi}^{(k 00)}= & -\frac{m_{e}}{\mu} \int_{0}^{1} d a a \int_{0}^{1} d b\left[-m_{e}(1-a) / \mu\right]^{k} \\
& \times \ln \left(\frac{D_{x \pi \pi}}{2 \mu m_{e}}\right),
\end{aligned}
$$

with

$$
\begin{aligned}
D_{x \pi \pi}= & a \mu^{2}+(1-a) m_{x}^{2}-a(1-a) m_{e}^{2} \\
& -a^{2} b(1-b) q^{2} .
\end{aligned}
$$

In our calculational procedure we use configuration space expressions to obtain sigma-terms. Therefore, we need to perform Fourier transforms in the loop integrals,

$$
S=\int \frac{d^{3} k}{(2 \pi)^{3}} e^{-i k \cdot x} \Pi
$$

with $x=\mu r$ e $k=q / \mu$.

Thus the configuration space equivalent for $\bar{\Pi}_{x \pi \pi}^{(k 00)}$ can be written as

$$
\begin{aligned}
\bar{S}_{x \pi \pi}^{(k 00)}= & \frac{1}{2 \pi x^{3}} \frac{m_{e}}{\mu} \int_{0}^{1} d a a\left[-m_{e}(1-a) / \mu\right]^{k} \\
& \times \int_{0}^{1} d b(1+\theta x) e^{-\theta x},
\end{aligned}
$$

where

$$
\theta^{2}=\phi^{2} /[b(1-b)]
$$

and

$$
\phi^{2}=\left[a \mu^{2}+(1-a) m_{x}^{2}-a(1-a) m_{e}^{2}\right] /\left(\mu^{2} a^{2}\right) .
$$

The above integral can acquire an imaginary part. This fact becomes evident when we perform the integral in the variable $b$,

$$
\begin{aligned}
\bar{S}_{x \pi \pi}^{(k 00)}= & \frac{1}{\pi x^{2}} \frac{m_{e}}{\mu} \int_{0}^{1} d a a\left[-m_{e}(1-a) / \mu\right]^{k}|\phi| \\
& \times\left\{\theta\left(\phi^{2}\right) K_{1}(2 \phi x)\right. \\
& \left.-\theta\left(-\phi^{2}\right) \frac{\pi}{2}\left[Y_{1}(2|\phi| x)-i J_{1}(2|\phi| x)\right]\right\} .
\end{aligned}
$$

In general, when $\left(\mu+m_{x}\right) \geq m_{e}$, the function $\phi^{2}$ is always positive. On the other hand, when $\left(\mu+m_{x}\right)<m_{e}$, there is an interval in the integration variable $a$ for which the function $\phi^{2}$ becomes negative. In case this happens, the functions $K_{\ell}$ must be replaced by $Y_{\ell}$. The former are monotonic functions, whereas the latter oscillate and produce contributions with alternating signs inside the integrals. This kind of behaviour is illustrated in Fig. 3, for the arbitrary choices $m_{e}=1150 \mathrm{MeV}$, $\mu=150 \mathrm{MeV}$. In this case the decay threshold corresponds to $m_{x}=1000 \mathrm{MeV}$.

This is the reason why the contributions in the case $(\mu+$ $\left.m_{x}\right)<m_{e}$, which represents the fact that the external particle can decay, tend to be small. As this is a general feature of the Feynman diagrams, it happens whenever an unstable particle is present. One must bear in mind, however, that the size of this effect depends on both the coupling constants of the specific problem and on the gap $m_{e}-m_{x}-\mu$. It is interesting to note that the importance of understanding the smallness of the delta sigma-term has been recently stressed in a conference talk by Meissner [12] and, to our knowledge, our paper is the only one proposing that the solution to this problem may be associated with the instability of the delta. 


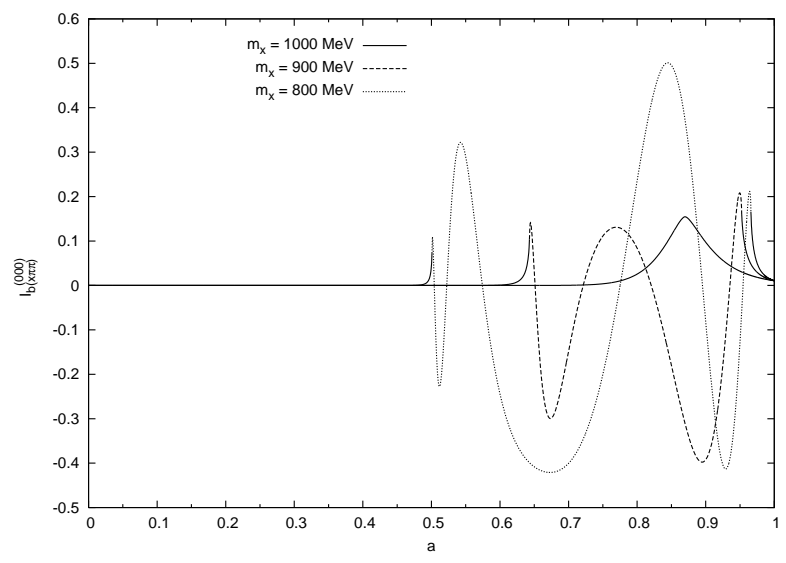

FIG. 3: Dependence in $a$ of the integrand of expression (16), with $m_{e}=1150 \mathrm{MeV}$ at $r=2.5 \mathrm{fm}$, for a choice of values of $m_{x}$. The dashed and dotted curves represent the intervals in which $\phi^{2}<0$.

\section{SUMMARY}

We have reviewed a model aimed at determining sigmaterms, which consists in cutting off configuration space expressions at the point where, as we assume, a phase transition occurs. Our main results are $43 \mathrm{MeV} \leq \sigma_{N} \leq 49 \mathrm{MeV}$ and $28 \mathrm{MeV} \leq \sigma_{\Delta} \leq 32 \mathrm{MeV}$, depending on the coupling constants employed. We have shown that the decay $\Delta \rightarrow \pi N$ changes the behaviour of loop integrals and gives rise to an oscillation in the cloud $N$ contribution to $\sigma_{\Delta}$ which is responsible for the relation $\sigma_{\Delta}<\sigma_{N}$.

\section{Acknowledgments}

This work was supported by CNPq, FAPESP and FUNDECT/MS (Brazilian agencies).
[1] T. R. Hemmert, B. R. Holstein, and J. Kambor, J. Phys. G 24, 1831 (1998).

[2] N. Kaiser, S. Gerstendörfer, and W. Weise, Nucl. Phys. A 637, 395 (1998); N. Fettes and Ulf-G. Meissner, Nucl. Phys. A 693, 693 (2001)

[3] H. Hellmann, Einführung in die Quantenchemie (Deuticke Verlag, Leipzig, 1937); R.P. Feynman, Phys. Rev. 56, 340 (1939); S. T. Epstein, Am. J. Phys. 22, 613 (1954);

[4] J. Gasser, H. Leutwyler, and M. E. Sainio, Phys. Lett. B 253 252 (1991); 253, 260 (1991).

[5] J. Gasser, M.E. Sainio, and A. Švarc, Nucl. Phys. B 307, 779 (1988).
[6] T. Becher and H. Leutwyler, Eur. Phys. Journal C 9, 643 (1999); JHEP 6, 17 (2001)

[7] I. P. Cavalcante, M. R. Robilotta, J. Sa Borges, D. de O. Santos, and G. R. S. Zarnauskas, Phys. Rev. C 72, 065207 (2005).

[8] M. R. Robilotta, Phys. Rev. C 63, 044004 (2001).

[9] C. Fronsdal, N. Cim. Suppl. 9, 416 (1958).

[10] V. E. Lyubovitskij, Th. Gutsche, A. Faessler, and E. G. Drukarev, Phys. Rev. D 63, 054026 (2001).

[11] V. Bernard, T. R. Hemmert, and Ulf-G. Meissner, Phys. Lett. B 622, 141 (2005).

[12] Ulf-G. Meissner, Proc. Sci. LATT2005, 009 (2005). 